\title{
Influence of Supporting Base Characteristics on Shrinkage-Induced Stresses in Concrete Pavements
}

\author{
By Jun Zhang' and Victor C. $\mathrm{Li}^{1}{ }^{2}$ Fellow, ASCE
}

\begin{abstract}
This paper presents an analytical model for prediction of shrinkage-induced stresses and displacements in concrete pavements due to the restraint of the supporting base. The model relies upon the interfacial friction stress transmitted between the slab and base as the fundamental mechanism of restraint provided by the base when concrete shrinks. By analyzing experimental results reported in the literature, a bilinear relation describing the friction stress-slippage behavior of the slab/base interface is adopted in the model. Closed-form expressions of shrinkage stresses and displacements in the concrete slab are obtained. The model quantifies the effects of concrete properties and the interfacial characteristics of the concrete slab and base on the shrinkage stresses and displacements. By applying the slab/base frictional stress-slippage relation of seven typical supporting bases in the model, the influences of the supporting base on the development of shrinkage stresses and displacements in the concrete slab are investigated. In addition, the effect of "bond breaker" layers on the shrinkage stresses in the concrete slab lying on a hot mix asphalt concrete base is studied with the model.
\end{abstract}

\section{INTRODUCTION}

Plain and steel reinforced cement concrete pavements have been widely used in modern transportation engineering due to their structural strength, desirable surface characteristics, durability, and economy. The average service life of a concrete pavement is determined by many factors including initial design, material properties, traffic, environment, salt application, presence and effectiveness of protective systems, and maintenance practices among others. All these factors influence the development of cracks in concrete slabs during service. Cracking and joint openings in pavements may further lead to concrete spalling and punchouts. In addition, cracks allow water and other chemical agents, such as deicing salt, to go through the cover layer and come into contact with the reinforcements, leading to reinforcement corrosion and rupture.

Concrete shrinkage and temperature changes may be the two major mechanisms leading to the initial crack formation and joint opening in concrete pavements. That is because, generally, pavements have a much larger surface area compared to other kinds of structural members, such as beams and columns. As a result, shrinkage-induced cracking in concrete pavements becomes more critical. Concrete shrinks as the cement paste hardens. The magnitude of shrinkage can be reduced by using concrete with the smallest possible amount of water and cement compatible with other requirements, such as strength and workability, and by moist-curing of sufficient duration. However, no matter what precautions are taken, a certain amount of shrinkage is usually unavoidable. If a concrete slab of moderate dimensions rests freely on its supports, it can contract to accommodate the shortening of its length produced by shrinkage. However, in pavements, the concrete slab and supporting base are in contact with each other and the slab cannot contract freely. Thus, as concrete shrinks, a certain amount of tensile stresses will be developed in the slab. The resulting tensile stresses in concrete due to shrinkage and restraints from connected members are known as shrinkage stresses. A change in temperature may have an effect similar

${ }^{1}$ Assoc. Prof., Dept. of Civ. Engrg., Tsinghua Univ., Beijing, 100084, PRC.

${ }^{2}$ Prof. and Dir., ACE-MRL, Dept. of Civ. and Envir. Engrg., Univ. of Michigan, Ann Arbor, MI.

Note. Discussion open until May 1, 2002. To extend the closing date one month, a written request must be filed with the ASCE Manager of Journals. The manuscript for this paper was submitted for review and possible publication on August 8, 2000; revised April 11, 2001. This paper is part of the Journal of Transportation Engineering, Vol. 127, No. 6, November/December, 2001. CASCE, ISSN 0733-947X/01/00060455-0462/\$8.00 + \$.50 per page. Paper No. 22389. to shrinkage, which also results in stresses in the pavement, with increasing temperature results in compressive stresses and decreasing temperature results in tensile stresses.

It should be clearly understood that stresses caused by concrete shrinkage and temperature changes are independent of applied loads. However, load stresses must be added to the restrained movement stresses, including thermal warping stress, to such an extent that the slab may crack. In some cases, the shrinkage- or temperature change-induced stresses are large enough to crack the slab.

Shrinkage of concrete, as a material property, has been extensively investigated in the past and has been understood well (Neville 1981; Mehta 1985). However, the investigations on shrinkage-induced stresses in concrete structures, such as concrete pavements, are still limited. For many years, pavement engineers have recognized the need for better models to predict pavement performance and to implement pavement design procedures (Moody 1998). The shrinkage-induced stress model obviously is one of these required models due to the previously described reasons. The developed model has to be able to predict the development of shrinkage stresses in the slab after casting. Since the shrinkage stresses in the pavement slab principally resulted from concrete shrinkage and restraint of the base, the model has to reflect the influences of concrete properties, supporting base properties and the slab/base interfacial friction characteristics.

In this paper, interfacial friction between slab and supporting base is recognized as the mechanism leading to stresses in slab as concrete shrinkage occurs. The problem is simplified as one dimension, and a bilinear friction stress-slippage relation is used. Analytic solutions of stress and displacement fields in the slab under shrinkage load are developed. Using the model as an analysis tool, the influence of the supporting base on the shrinkage-induced stresses and displacements in the concrete slab are predicted and discussed. A total of seven base types that are commonly applied in pavements are investigated. Model predictions clearly indicate that the influence of base characteristics on the shrinkage stresses and displacements in pavement slab is quite significant. This influence has to be considered in concrete pavement design. In addition, performance of concrete pavements may be improved by slab/ base interface tailoring. The effect of "bond breaker" layers on the shrinkage stresses in the concrete slab on a hot mix asphalt concrete (HMAC) base is studied with the model.

\section{DEVELOPMENT OF MODEL}

To analyze the response of concrete pavements under shrinkage strain, certain assumptions have to be made prior to 
formulation of the mathematical model. These assumptions are as follows:

- The concrete behaves in a linearly elastic manner.

- All shrinkage resulted deformation is uniformly distributed throughout the concrete slab.

- The base beneath the concrete slab is a rigid material and the deformation under action of the horizontal friction forces is ignored.

- The effects of warpage and creep of concrete are ignored.

The uniformly distributed shrinkage strain in the slab support simulating stress development along slab length is sufficient to reflect the magnitude of stresses in the slab. This consideration allows the mathematical model to be considered a $1 \mathrm{D}$ problem. A concrete pavement section with width $B$, height $H$, and length $L$ is modeled, as shown in Fig. $1 ; y$ is the direction perpendicular to the slab length, and $x$ is the direction parallel to the slab length. The ends of the slab are located at $x=0$ and $x=L$, respectively. Due to the symmetry of the problem, only half of the slab is modeled.

Before derivation of the governing equations for the model, a law governing the slab/base interfacial friction stress-slippage relation needs to be given. A number of experimental studies had shown that the development of slab/base interfacial friction stress with slab slippage can be described by a bilinear function (Stott 1961; Wesevich et al. 1987; Wimsatt et al. 1987; Rozycki and Rasmussen 1998). Typical test results of concrete slab sliding on different supporting bases in terms of friction stress versus slab displacement diagrams are shown in Fig. 2. From these test results, it can be seen that the shear stress first almost linearly increases to a certain value (the so-

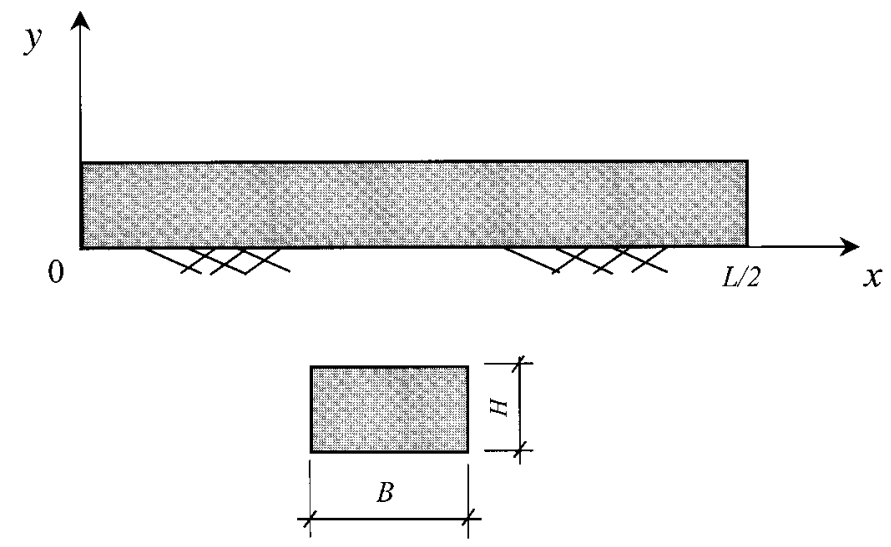

FIG. 1. Schematic Diagrams Showing Slab Model Used in Analyses

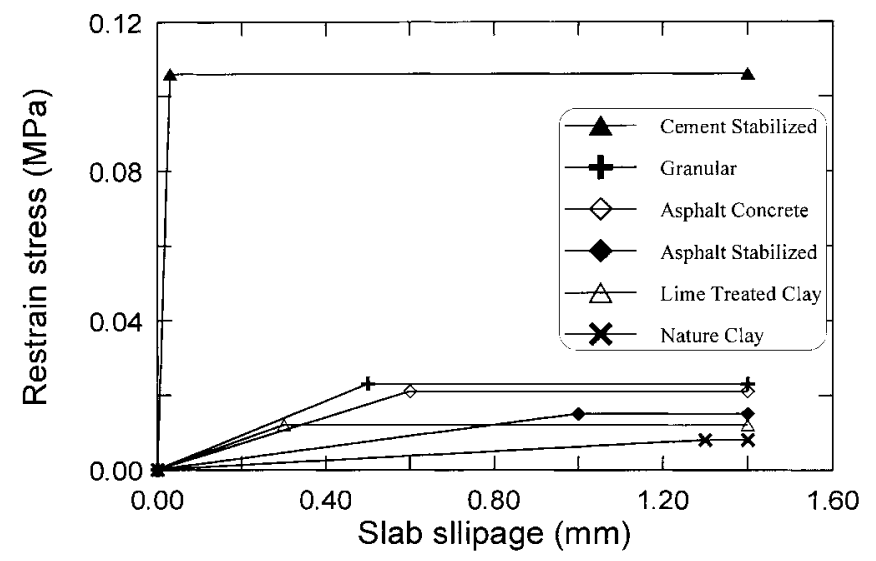

FIG. 2. Typical Experimentally Determined Restrain Stress of Supporting Base as Function of Slab Slippage called steady-state value) with the increase of slab slippage. Then the stress stays at this value with further increase of the slab slippage. However, it should be noted that the frictional stress-slippage relationship may not follow the classical Coulomb friction law because the mechanisms of slab/base friction do not only include the shear sliding, but also include adhesion and bearing between slab and base (Wesevich et al. 1987). Some experimental results show that slab thickness does not influence the steady frictional stress and some show that the steady frictional stress increases with slab thickness (Wesevich et al. 1987). It may depend upon the component that dominates the slab/base friction. Further investigations on the influence of slab thickness on the friction stress between slab and supporting base are necessary. In the present work, friction stress acting on the slab surface as modeled as

$$
\begin{gathered}
\tau=\frac{\tau_{0}}{\delta_{0}}|u| \text { for } 0 \leq|u| \leq \delta_{0} \\
\tau=\tau_{0} \quad \text { for } \quad \delta_{0}<|u|
\end{gathered}
$$

where $u=$ average slippage along thickness of the slab relative to base at location $x$, which is given by

$$
u(x)=u_{\varepsilon}(x)+u_{c}(x)
$$

where $u_{\varepsilon}=$ displacement induced by concrete shrinkage; $u_{c}=$ displacement resulting from internal axial stress; $\tau_{0}=$ steadystate frictional stress; and $\delta_{0}=$ corresponding slippage as the frictional stress achieves $\tau_{0}$. The restraint characteristics $\tau_{0}$ and $\delta_{0}$ are normally governed by the type of base, and they can be determined by sliding tests with a laboratory size slab cast on a given base. The frictional shear stress model given by (1) is schematically shown in Fig. 3.

When the slab is subjected to a shrinkage strain $\varepsilon_{c}$ in the direction of slab length, based on the above assumptions, the axial force equilibrium for a slab length $d x$ in the presence of a thickness average axial stress $\sigma_{c}$ and slab/base interfacial shear stress $\tau$ requires

$$
\frac{\partial \sigma_{c}}{\partial x}-\frac{\tau}{H}=0
$$

From elasticity theory, the first derivative of $\sigma_{c}$ with respect to $x$ can be related to axial displacement $u_{c}$ by

$$
\frac{\partial \sigma_{c}}{\partial x}=E_{c} \frac{\partial^{2} u_{c}}{\partial^{2} x}
$$

where $E_{c}=$ Young's modulus of concrete. Replacing $\partial \sigma_{c} / \partial x$ with (4) in (3) and noting that

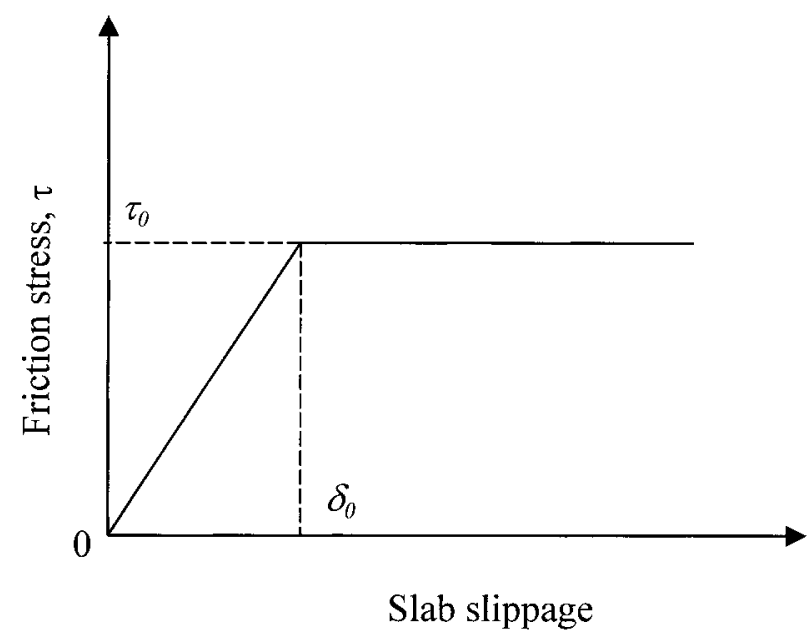

FIG. 3. Friction Stress-Slab Slippage Relationship Used in Model 


$$
\frac{\partial^{2} u}{\partial^{2} x}=\frac{\partial^{2} u_{c}}{\partial^{2} x}, \quad\left(\frac{\partial^{2} u_{\varepsilon}}{\partial^{2} x}=0\right)
$$

the general equation governing the average axial displacement distribution in the slab $u$ is

$$
\frac{\partial^{2} u}{\partial^{2} x}-\frac{\tau}{E_{c} H}=0
$$

According to the magnitude of displacement, the slab may finally be divided into two sections on each of which a specific $\tau$ should be applied. The detailed derivations of stress and displacement fields for a given shrinkage strain are given as follows.

\section{Stage I: $|u| \leq \delta_{0}$ at $x=0$}

In this case, the slab/base interfacial friction stress $\tau$ linearly increases with the increase of slab slippage $u$, and this linear friction stress-slippage relationship can be applied along the whole slab. Replacing $\tau$ with the linear relation given by (1) in (4), one has

$$
\frac{\partial^{2} u}{\partial^{2} x}-\frac{\tau_{0}}{E_{c} H \delta_{0}} u=0
$$

To solve this differential equation with boundary conditions in which $u=0$ at $x=L / 2$ and $\partial u / \partial x=\left(\sigma_{c 0} / E_{c}\right)+\varepsilon_{c}$ at $x=0$, yields

$$
u=-\frac{1}{\beta}\left(\frac{\sigma_{c 0}}{E_{c}}+\varepsilon_{c}\right) \frac{\sinh \beta\left(\frac{L}{2}-x\right)}{\cosh \beta \frac{L}{2}}
$$

where $\beta=\sqrt{\tau_{0} / E_{c} H \delta_{0}}$; and $\sigma_{c 0}=$ average axial stress at $x=$ 0 along slab thickness. Here, $\sigma_{c 0}$ is defined as negative if it compresses the slab and positive if it tensions the slab. Similarly, $\varepsilon_{c}$ is negative if it makes the slab shorter and positive if it makes the slab longer. In the present study (shrinkage strain is considered only), $\varepsilon_{c}$ should always be a negative value. The sign of $u$ is governed by the combined effect of $\varepsilon_{c}$ and $\sigma_{c 0}$. Positive $u$ means that the slab becomes shorter, and negative value means that the slab becomes longer. In fact, the slab displacement $u$ is always positive as only concrete shrinkage is considered. This means that the value of $\left(\sigma_{c 0} / E_{c}\right)+\varepsilon_{c}$ is always a negative value. From (2) the stress field $\sigma_{c}$ can be expressed as

$$
\sigma_{c}=E_{c}\left(\frac{\partial u}{\partial x}-\varepsilon_{c}\right)
$$

Substitution of $u$ with (7) into (8) gives

$$
\sigma_{c}=-E_{c} \varepsilon_{c}\left[1-\frac{\cosh \beta\left(\frac{L}{2}-x\right)}{\cosh \beta \frac{L}{2}}\right]+\sigma_{c 0} \frac{\cosh \beta\left(\frac{L}{2}-x\right)}{\cosh \beta \frac{L}{2}}
$$

It should be noted that the sign of $\sigma_{c}$ has the same meanings as $\sigma_{c 0}$.

From (7), it can be seen that at this stage the maximum displacement of slab $u_{m}$ occurs at $x=0$, i.e.,

$$
u_{m}=-\frac{1}{\beta}\left(\frac{\sigma_{c 0}}{E_{c}}+\varepsilon_{c}\right) \tanh \beta \frac{L}{2}
$$

In jointed concrete pavements, $2 u_{m}$ is equal to the joint opening. When $d \sigma_{c} / d x=0$, one obtains $x=L / 2$, and so the maximum average stress in slab $\sigma_{c m}$ occurs at this location, and it is given by

$$
\sigma_{c m}=-E_{c} \varepsilon_{c}\left[1-\frac{1}{\cosh \beta \frac{L}{2}}\right]+\sigma_{c 0} \frac{1}{\cosh \beta \frac{L}{2}}
$$

Eqs. (10) and (11) provide the general relationships between maximum slab slippage, maximum stress, and concrete shrinkage strain for a given slab geometry and supporting base characteristics in the case of $|u| \leq \delta_{0}$ at $x=0$.

\section{Stage II: $|u|>\delta_{0}$ at $x=0$}

In this case, the slab should be divided into two sections according to the displacement relative to the base. Now assuming that $u=\delta_{0}$ at $x=x_{0}$, for the section of $0 \leq x \leq x_{0}$, the slab/base interfacial friction stress is fully developed and the shear stress is a constant $\tau_{0}$, as shown in (1). Replace $\tau$ with $\tau_{0}$ in (4), the general governing equation of $u$ can be rewritten

$$
\frac{\partial^{2} u}{\partial^{2} x}-\frac{\tau_{0}}{E_{c} H}=0
$$

To solve this differential equation with boundary conditions in which $\partial u / \partial x=\left(\sigma_{c 0} / E_{c}\right)+\varepsilon_{c}$ at $x=0$ and $u=\delta_{0}$ at $x=x_{0}$ yields

$$
u=\delta_{0}+\frac{1}{2} \beta^{2} \delta_{0}\left(x^{2}-x_{0}^{2}\right)+\left(\frac{\sigma_{c 0}}{E_{c}}+\varepsilon_{c}\right)\left(x-x_{0}\right)
$$

Here $\varepsilon_{c}$ and $\sigma_{c 0}$ have the same meanings as described previously regarding their negative or positive signs. Similar to stage I, using (13) in (8), the stress in the slab within this location can be expressed as

$$
\sigma_{c}=E_{c} \beta^{2} \delta_{0} x+\sigma_{c 0}
$$

For the section of $x_{0}<x \leq L / 2$, the slab/base frictional stress is being developed due to the small slab slippage $\left(u \leq \delta_{0}\right)$. The procedures used to solve the average displacement and stress fields in stage I can be applied in this section by simply replacing the slab length $L / 2$ with $\left(L / 2-x_{0}\right)$ and using a new boundary condition at $x=x_{0}$ instead of at $x=0$ [i.e., $\partial u / \partial x=$ $\left(\sigma_{c 00} / E_{c}\right)+\varepsilon_{c}$ at $x=x_{0}$, where $\sigma_{c 00}$ is the thickness average axial stress at $x=x_{0}$ ]. The displacement and stress fields in this section are given by

$$
u=-\frac{1}{\beta}\left(\frac{\sigma_{c 00}}{E_{c}}+\varepsilon_{c}\right) \frac{\sinh \beta\left(\frac{L}{2}-x\right)}{\cosh \beta\left(\frac{L}{2}-x_{0}\right)}
$$

$\sigma_{c}=-E_{c} \varepsilon_{c}\left[1-\frac{\cosh \beta\left(\frac{L}{2}-x\right)}{\cosh \beta\left(\frac{L}{2}-x_{0}\right)}\right]+\sigma_{c 00} \frac{\cosh \beta\left(\frac{L}{2}-x\right)}{\cosh \beta\left(\frac{L}{2}-x_{0}\right)}$

where

$$
\sigma_{c 00}=E_{c} \beta^{2} \delta_{0} x_{0}+\sigma_{c 0}
$$

The length $x_{0}$ can be numerically determined from (15) by setting $x=x_{0}$ and $u=\delta_{0}$, i.e.,

$$
\delta_{0}=-\frac{1}{\beta}\left(\beta^{2} \delta_{0} x_{0}+\frac{\sigma_{c 0}}{E_{c}}+\varepsilon_{c}\right) \tanh \beta\left(\frac{L}{2}-x_{0}\right)
$$


TABLE 1. Frictional Restraint Characteristics of Seven Typical Bases

\begin{tabular}{lcc}
\hline \hline Base type & $\begin{array}{c}\tau_{0} \\
(\mathrm{MPa})\end{array}$ & $\begin{array}{c}\delta_{0} \\
(\mathrm{~mm})\end{array}$ \\
\hline Cement stabilized (CS) & 0.106 & 0.025 \\
Untreated 32-mm HMAC (AC-R) & 0.104 & 0.25 \\
Granular (GR) & 0.023 & 0.50 \\
Asphalt concrete (AC-S) & 0.021 & 0.60 \\
Asphalt stabilized (AS) & 0.015 & 1.00 \\
Lime-treated clay (LTC) & 0.012 & 0.30 \\
Natural clay (NC) & 0.008 & 1.30 \\
\hline \hline
\end{tabular}

The above equation indicates that the length of the fully developed zone of the slab/base friction stress $x_{0}$ and the length of being development zone $\left(L / 2-x_{0}\right)$, are governed by the combined effects of $\delta_{0}, \beta, \sigma_{c 0}$, and $\varepsilon_{c}$.

The maximum displacement of slab $u_{m}$ at this stage can be obtained by setting $x=0$ in (13) i.e.,

$$
u_{m}=\delta_{0}\left(1-\frac{1}{2} \beta^{2} x_{0}^{2}\right)-\left(\frac{\sigma_{c 0}}{E_{c}}+\varepsilon_{c}\right) x_{0}
$$

When $d \sigma_{c} / d x=0$, from (14), one obtains $x=L / 2$. Thus the maximum thickness average stress $\sigma_{c m}$ at this stage occurs at this location, and it is given by

$$
\begin{gathered}
\sigma_{c m}=-E_{c} \varepsilon_{c}\left[1-\frac{1}{\cosh \beta\left(\frac{L}{2}-x_{0}\right)}\right] \\
+\left(E_{c} \beta^{2} \delta_{0} x_{0}+\sigma_{c 0}\right) \frac{1}{\cosh \beta\left(\frac{L}{2}-x_{0}\right)}
\end{gathered}
$$

Eqs. (19) and (20) provide the general relationships between the maximum slab movement, stress, and concrete shrinkage strain for a given slab geometry and base characteristics in the case of $|u|>\delta_{0}$ at $x=0$. In the design of jointed concrete pavements, values of $u_{m}$ and $\sigma_{c m}$ need to be controlled in order to limit the joint opening and to reduce the possibility of cracking in slab.

Eqs. (10) and (11) or (19) and (20) may serve as theoretical tools for slab length design in jointed concrete pavements based on the requirements of maximum tensile stress in the slab and maximum joint opening. It should be noted that, in design, temperature stress and the stress resulting from traffic load have to be incorporated along with the shrinkage stresses discussed in this paper. In addition, the current model may also serve as a tool for early age cracking control in concrete pavements. The cracking that resulted from shrinkage in concrete pavements is normally more critical in the first $72 \mathrm{~h}$ after construction than afterward due to the low tensile strength of concrete at this age. Furthermore, the stress resulting from uniform temperature changes can easily be incorporated into the model by replacing shrinkage strain $\varepsilon_{c}$ with $\varepsilon_{c}+\alpha \Delta T$, where $\alpha$ is the thermal coefficient of concrete material. When the temperature increases, $\Delta T$ is positive and compressive stresses should result in the slab. In contrast, when the temperature decreases, $\Delta T$ is negative and tensile stresses should be induced in the slab. The detailed discussions on the temperature stresses in the slab are out of the scope of the present study.

The stress and displacement distributions along the slab length given by (13), (14) and (15), (16) are schematically shown in Fig. 4. In general, $\sigma_{c 0}$ should be related with crack bridging law of concrete material if the slab ends are associated with crack location. The present work focuses on analyzing the development of shrinkage stress in jointed concrete pavements, so that $\sigma_{c 0}=0$ is used in the following analyses. It should be noted that the stress given by (9) or (14) and (16) is the stress averaged over slab thickness at location $x$. In re-

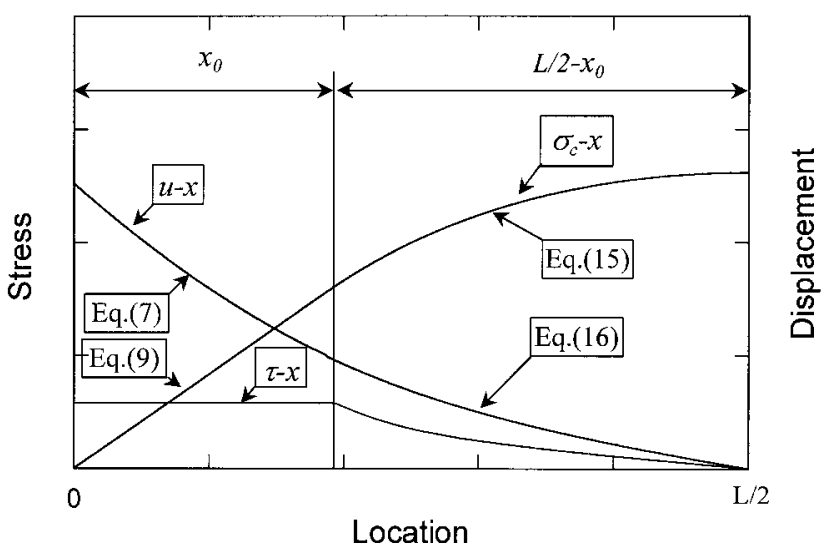

FIG. 4. Schematic Representation of Shrinkage Stress and Displacement Distributions along Half-Slab Length

ality, the shrinkage stress at a location close to the slab bottom should be greater than that close to the top due to the reduction of shear stress along the slab thickness. This means that even when the average tensile stress resulting in the slab along the whole thickness is smaller than that of the tensile strength of concrete, the slab may still be cracked due to the maximum tensile stress at the slab bottom exceeding the tensile strength of concrete. The crack may not go through the whole thickness, but it does exist and it may gradually propagate and finally reach the slab top. In addition, cracking possibility should also be influenced by the tensile strength of concrete material, which is a time-dependent material parameter.

The present analysis considers only shrinkage restraint from slab support. When other restraints such as reinforcing bars are present, they must also be accounted for in generating tensile shrinkage stresses in the slabs.

\section{RESULTS AND DISCUSSION}

\section{Material Parameters for Model Inputs}

As concrete material properties, the free shrinkage strain $\varepsilon_{c}$, elastic modulus $E_{c}$, and tensile strength $\sigma_{c t}$ are time-dependent. Since cracking in the matrix is a combined result of these parameters, shrinkage crack development also becomes timedependent. As an example, the following empirical expressions, which describe the time-dependent law of above parameters, are used in the present analysis.

Long-term studies (Branson 1977) show that, for moisturecured concrete at any time $t$ (in days), shrinkage can be predicted satisfactorily by

$$
\varepsilon_{c, t}=\frac{t}{35+t} \varepsilon_{c, u}
$$

where $\varepsilon_{c, t}=$ free shrinkage strain at time $t$ (in days); and $\varepsilon_{c, u}$ $=$ ultimate value after a long period of time. The time-dependent law of elastic modulus of concrete can be estimated by (Mosley and Bungey 1990)

$$
\begin{gathered}
E_{c, t}=E_{c, 28}[0.52+0.15 \log (t)] \text { for } t \leq 28 \\
E_{c, t}=1.019 E_{c, 28} \text { for } t>28
\end{gathered}
$$

where $E_{c, t}$ and $E_{c, 28}=$ elastic moduli at time $t$ (in days) and 28 days, respectively. In the following analyses, $\varepsilon_{c, u}$ and $E_{c, 28}$ equal to $8 \times 10^{-4}$ and $30 \mathrm{GPa}$ are assumed, respectively.

The rest of the important material parameters are the constants used in (1) to describe the slab/base interfacial friction stress-slippage relation. In the past, a number of experimental studies on evaluation of friction stress between the concrete slab and supporting bases had been carried out (Stott 1961; Wesevich et al. 1987; Wimsatt et al. 1987; Rozycki and Ras- 


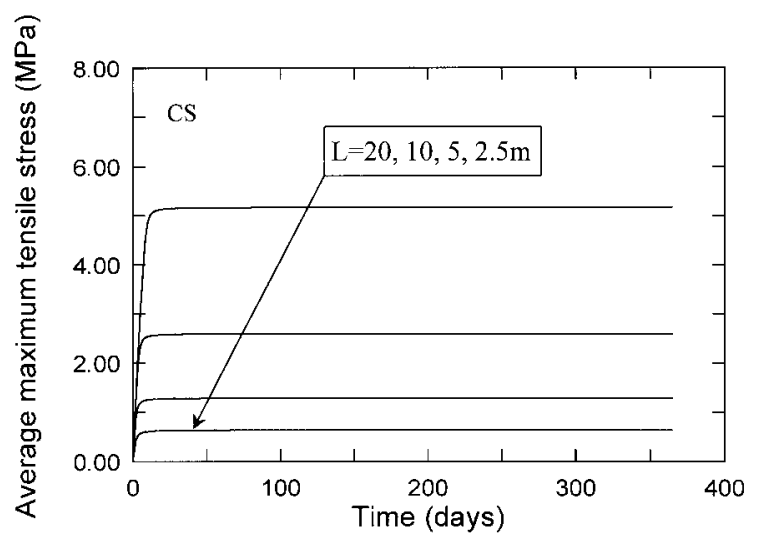

(a)

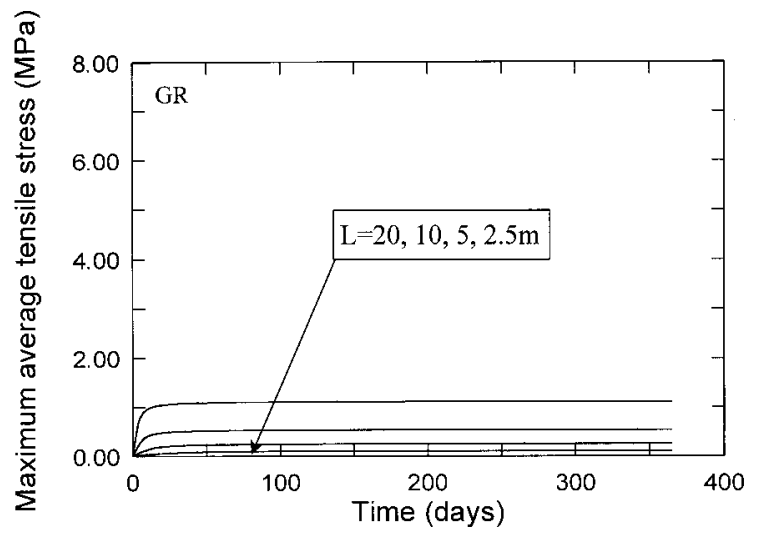

(c)

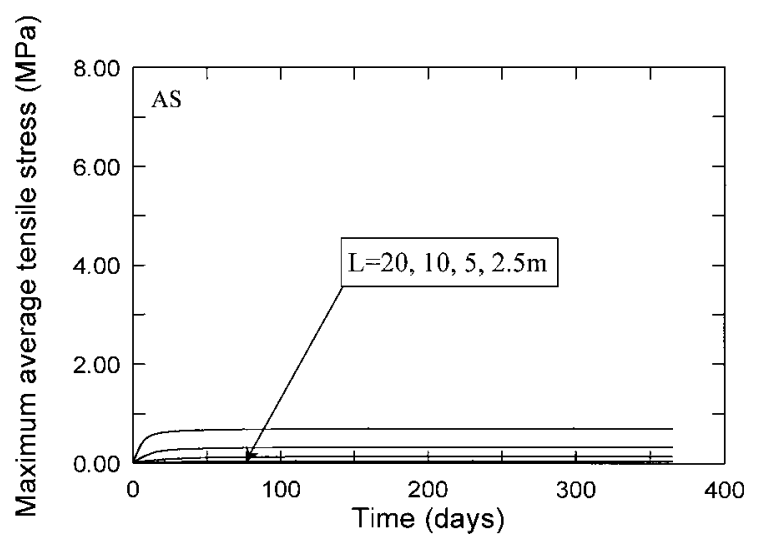

(e)

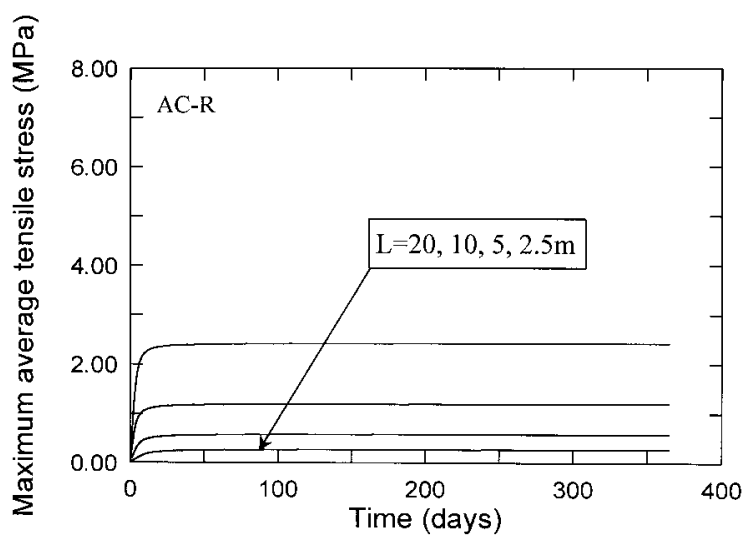

(b)

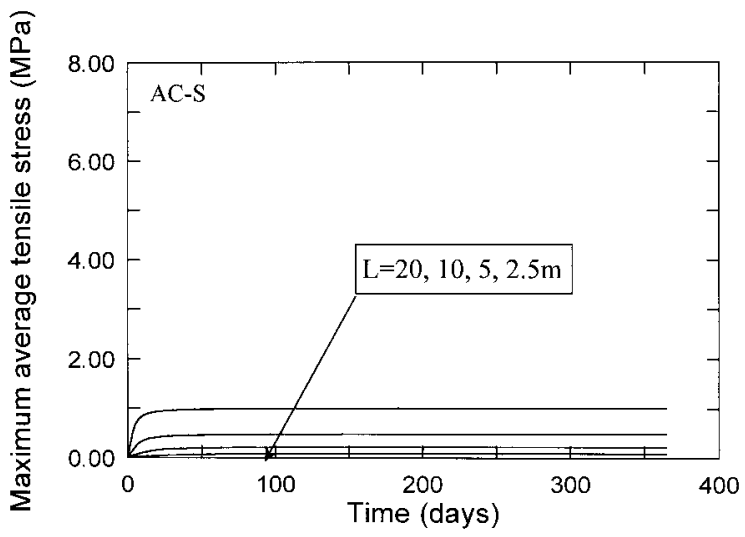

(d)

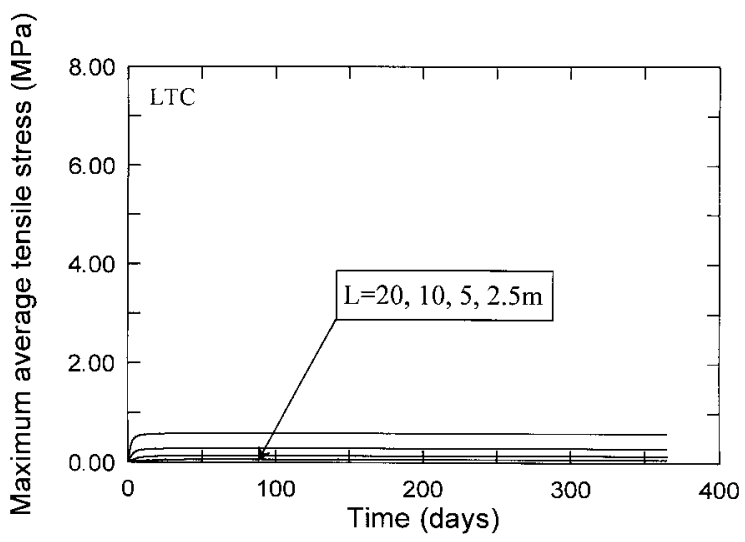

(f)

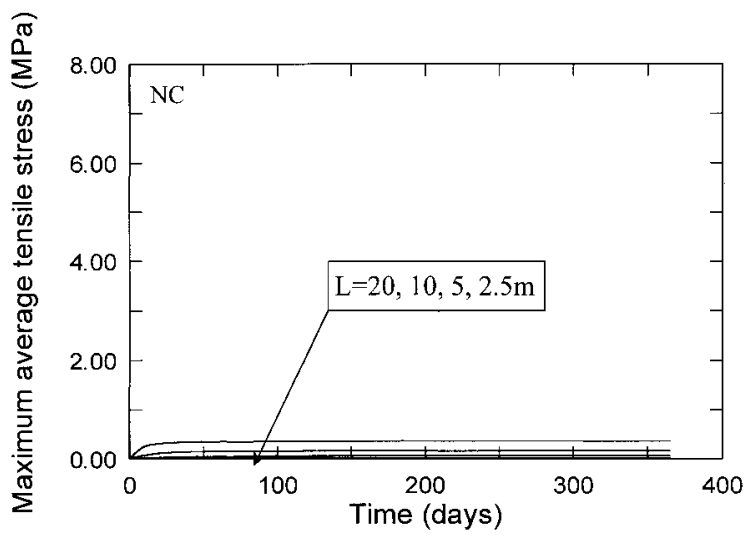

(g)

FIG. 5. Maximum Shrinkage Stresses in Concrete Pavement Slab as Function of Time after Slab Casting, Showing Predictions of Seven Typical Bases 


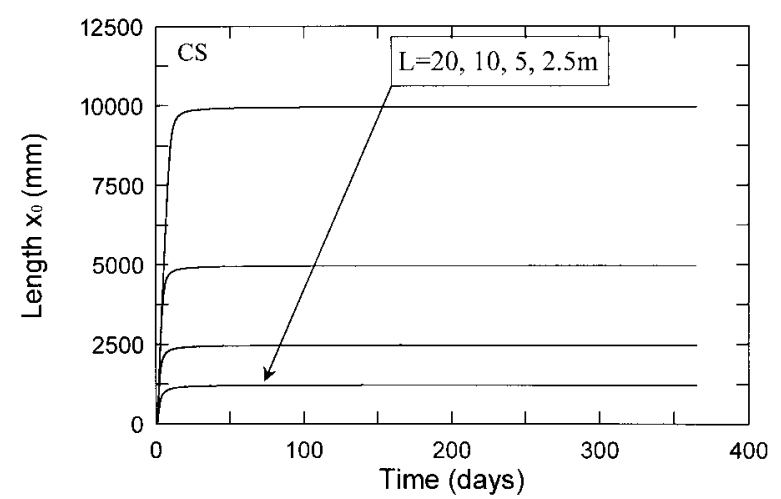

(a)

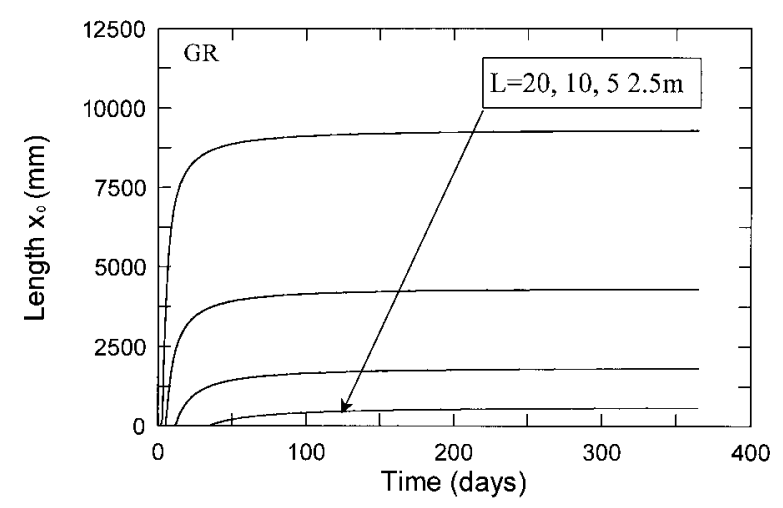

(c)

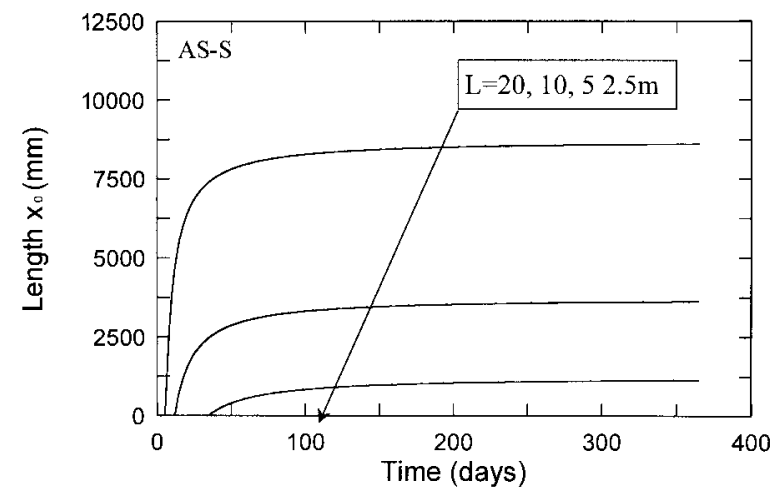

(e)

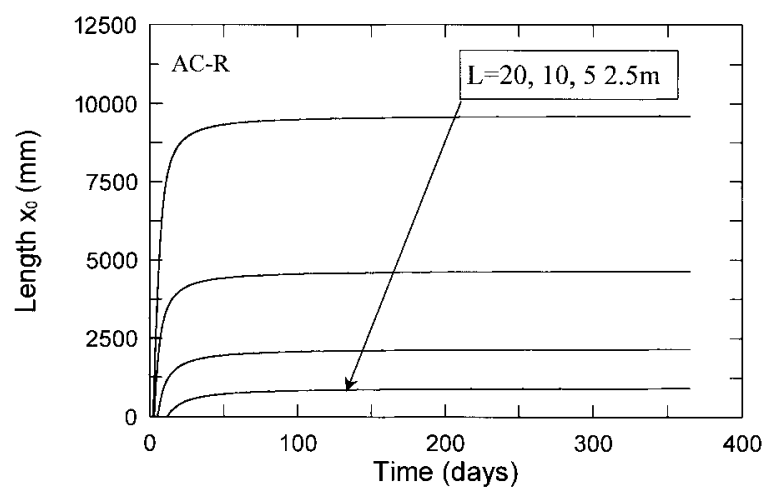

(b)

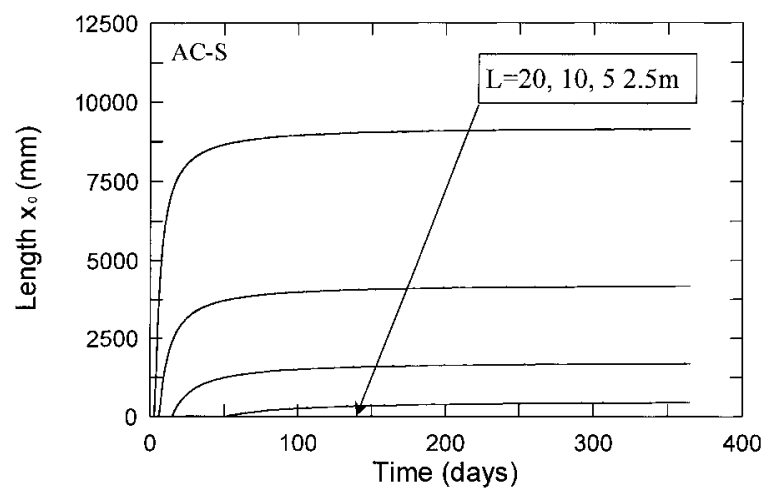

(d)

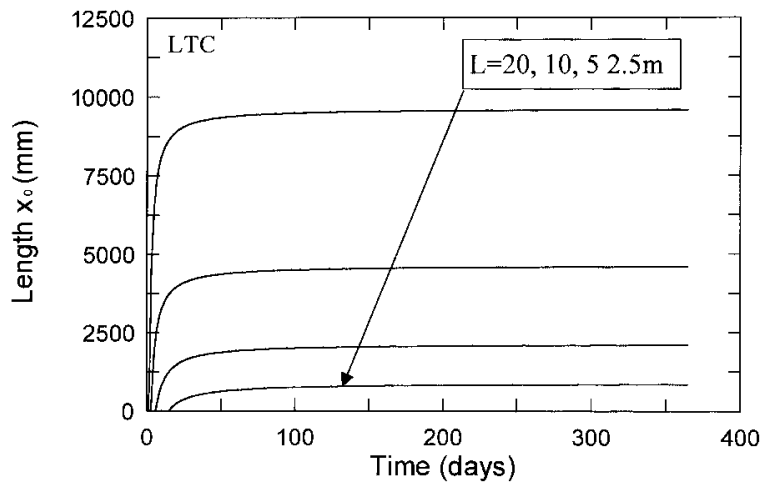

(f)

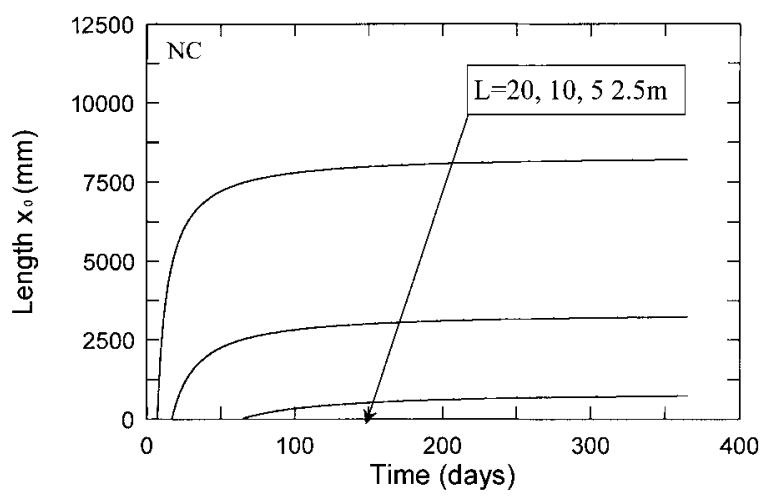

(g)

FIG. 6. Length $x_{0}$ as Function of Time Since Slab Casting, Showing Model Predictions of Seven Typical Bases

mussen 1998). Based on the experimental results (Wimsatt et al. 1987; Rozycki et al. 1998), the related parameters used in (1) for a number of typical supporting bases are determined and listed in Table 1.

\section{Model Predictions and Discussion}

As an example of calculation, a slab with $200 \mathrm{~mm}$ thickness lying on a given base is simulated. The types of bases used in 
the analyses are given in Table 1 . The influences of base characteristics on the shrinkage-induced stress as well as corresponding slab displacements are predicted using the above analytical model. The model results are shown in Figs. 5-9.

The average maximum concrete tensile stress $\sigma_{c m}$ as a function of time $t$ since casting, with four different slab lengths of 2.5, 5, 10, and $20 \mathrm{~m}$, is shown in Figs. 5(a-g). Corresponding value of $x_{0}$ as a function of time $t$ is shown in Fig. 6(a-g). From these model predictions, first, it can be seen that shrink-

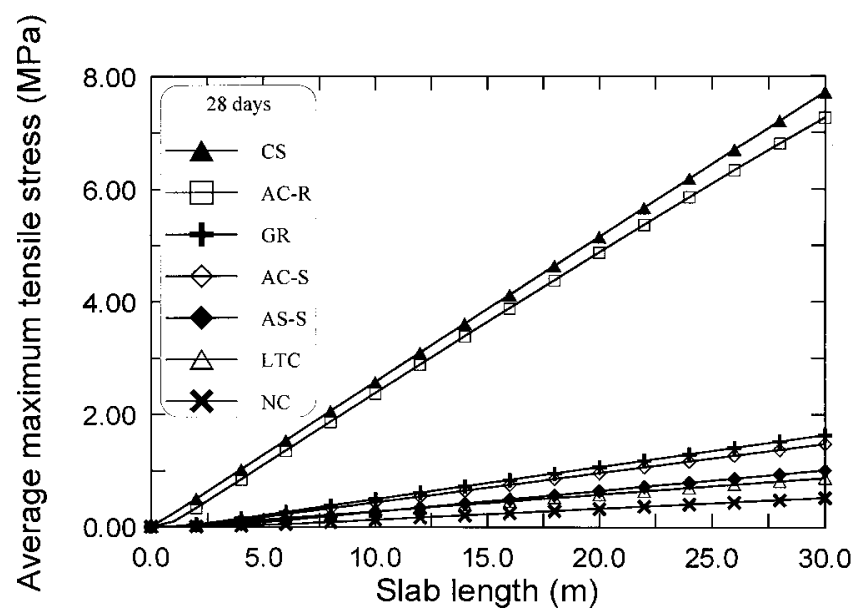

FIG. 7. Influence of Slab Length on Shrinkage Stresses in Concrete, Showing Model Predictions of Seven Typical Bases

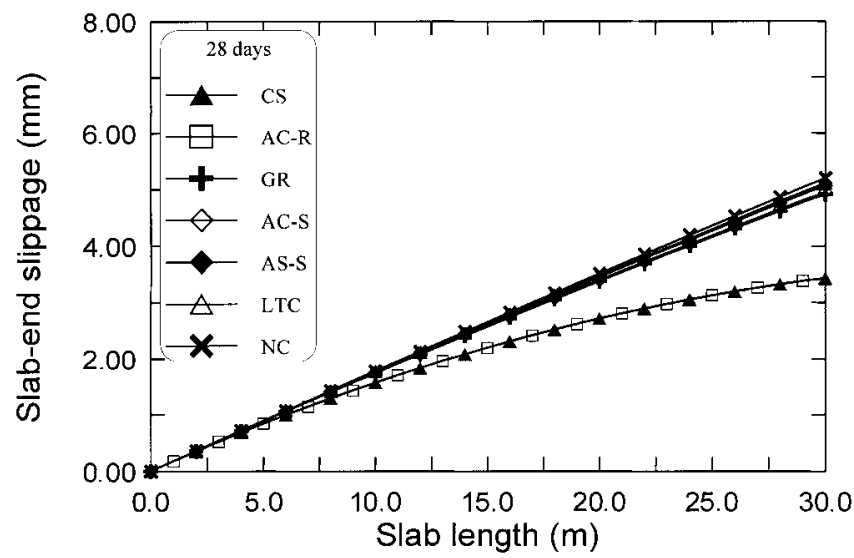

FIG. 8. Influence of Slab Length on Slab-End Displacements, Showing Model Predictions of Seven Typical Bases

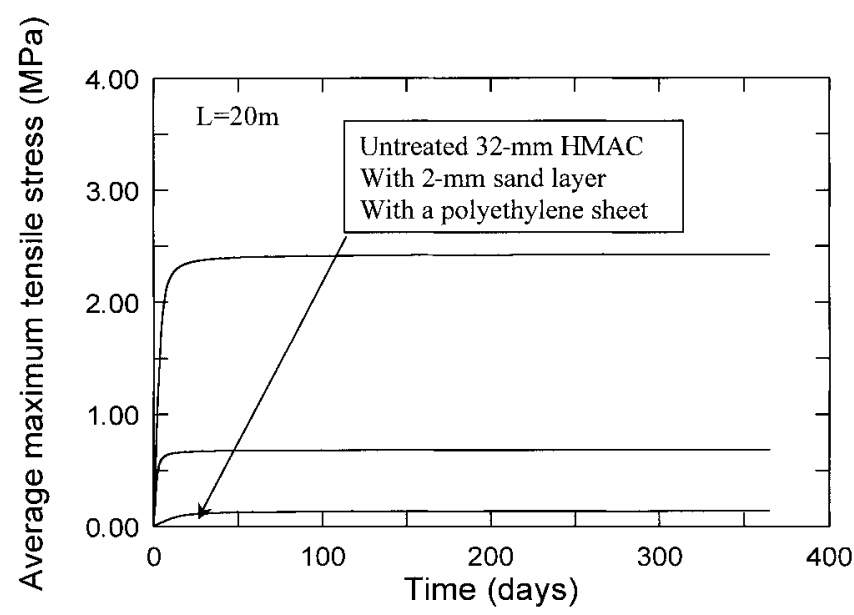

FIG. 9. Influence of Slab/Base Bond Breaker on Maximum Shrinkage Stresses in Concrete Slab, Showing Three Typical Modifications on Slab/ Base Interface age stress increases with time since casting. The development of the stress with time can be described by two stages. In the first stage, the stress increases with time at a high rate up to a certain value. In the second stage, it increases with time at a very low rate, almost represented as a constant stress in the figures. The stress development with time is quite consistent with the development of length $x_{0}$ with time [Figs. 6(a-g)]. As described before, $x_{0}$ is governed by the combined effects of $\delta_{0}, \beta$, and $\sigma_{c 0}$ for a given shrinkage strain $\varepsilon_{c}$. Graphically, it can be seen that the upper limitation of $x_{0}$ is $L / 2$. Therefore, from (20), the maximum shrinkage stress is equal to $0.5 \tau_{0} L / H$ $+\sigma_{c 0}$ in the case of $|u|>\delta_{0}$ at $x=0$. It is also noted that the transition point from stage I to stage II is different for different base cases. This transition point depends upon the parameters of $\delta_{0}, \beta$, and $\sigma_{c 0}$. In the case of $\sigma_{c 0}=0$ (stress free at slab ends), this point is only governed by the slab/base interfacial shear stress characteristics.

By comparing the magnitudes of the stresses in different base cases, it can be concluded that the type of bases significantly influences the shrinkage stresses in the concrete slab. The difference between the maximum and the minimum ones, such as for cement stabilized base and nature clay base, can achieve several megapascals under the same concrete shrinkage strain [Figs. 5(a and g)]. However, this does not mean that the cement-stabilized base is worse than the nature clay base in the view of overall structural performance of concrete pavements. This is because structural performance depends not only on the shrinkage stress level, but also on many other factors, such as load transfer ability, slab deflection, and so forth. But modifications on the slab/base interface may be needed to reduce the shrinkage stress in the slab.

Second, as expected, the shrinkage stresses are also a function of slab length. Fig. 7 shows the relationship between maximum shrinkage stress and slab length with different bases. The stress increases with an increase of slab length. The higher the steady-state friction stress $\tau_{0}$, the higher the rate of stress increase with slab length. The slab-end slippage is also a function of slab length. Fig. 8 shows the influence of slab length on the slab-end displacements, where the results with different supporting bases are present together. It can be seen that base characteristics can also influence the joint opening in concrete pavement. Generally, the longer the slab, the greater the slabend movement. The higher the slab/base shear stress, the lower the slab-end displacement. Due to the compensation of tensile deformation to overall displacement of slab, the relationship of slab-end displacement and slab length $u_{m}-L$ gradually becomes nonlinear in the case of high slab/base friction stress. In addition, the difference between the slab-end displacements of different supporting bases is also a function of slab length due to the difference in tensile deformation in the slab. The longer the slab, the larger the difference of the slab-end displacement.

From the above results one can conclude that frictional restraint from supporting base may result in high enough tensile stresses to crack concrete slabs. The results also indicate that a stiff base, such as the cement-treated base may result in high frictional resistance, even though the mechanism of the frictional resistance between the slab and supporting base needs to be further investigated in the future. However, in order to transfer traffic load effectively without excessive slab deflections, a relatively stiff base is necessary in concrete pavement structures. The high frictional resistance may need to be decreased in order to lower the possibility of slab cracking in normal concrete pavements and/or to reduce stress lost in prestressed concrete pavements. Rozycki and Rasmussen (1998) carried out an experimental study on modifying the interfacial properties of concrete slab and HMAC base. The modification methods and corresponding parameters describing the slab/ 
TABLE 2. Restraint Characteristics of HMAC Base (32-mm Maximum Aggregate Size) Results before and after Modifications on Slab/Base Interface

\begin{tabular}{lcc}
\hline \hline Base type & $\begin{array}{c}\tau_{0} \\
(\mathrm{MPa})\end{array}$ & $\begin{array}{c}\delta_{0} \\
(\mathrm{~mm})\end{array}$ \\
\hline Untreated 32-mm HMAC & 0.104 & 0.25 \\
Untreated 32-mm HMAC with 2-mm sand & 0.023 & 0.50 \\
Untreated 32-mm HMAC with polyethylene sheet & 0.007 & 0.60 \\
\hline \hline
\end{tabular}

base frictional stress-slippage relation are summarized in Table 2. Applying the parameters list in Table 2 as model inputs, the development of shrinkage stresses in the slab in terms of maximum tensile stress and time diagram $\sigma_{c m}-t$ are presented in Fig. 9. From the model predictions one can easily see that the shrinkage-induced stresses in the concrete slab are greatly reduced by applying a 2-mm sand layer or polyethylene sheets between the slab and HMAC base. These results indicate that, in practice, it is possible to modify the slab/base interfacial properties to achieve a prescribed stress state in slabs.

In addition, it is well known that the design of slab length in jointed concrete pavements is normally based on two criteria. One is to limit the stress level to prevent cracking in the slab. The stress should be the sum of all possible stresses induced in the slab, such as shrinkage- and temperature changeinduced stresses including curling stresses and stresses due to traffic load. Another is to limit the maximum joint opening. Because overly large joint opening may result in concrete spalling and lose efficiency of sealant material, which leads to the infiltration of surface water into subbase and foundation. This water may lead to severe pumping, faulting, loss of support, and slab breakup. Yoder and Witczak (1975) pointed out that joints without dowels are generally satisfactory if the joint opening is $1.02 \mathrm{~mm}$ or less. For doweled joints the opening should be $6.35 \mathrm{~mm}$ or less (Yoder and Witczak 1975). The designed slab length in jointed concrete pavements should be the smaller one of the lengths required according to the strength and joint opening criterion. From Figs. 7 and 8, one can see for a given concrete tensile strength, or a given joint opening $2 u_{m}$, the required slab length is significantly influenced by the base characteristics, although only shrinkage stress is considered. The developed model may serve as a tool for slab length design and be used for improving the overall performance of concrete pavements.

\section{CONCLUSIONS}

This paper presented an analytic model for prediction of shrinkage stresses in concrete pavements due to the restraint of the supporting base. The model is based on recognizing that the interfacial friction stress between slab and supporting base is the mechanism leading to stress in the slab as the concrete shrinks. By analyzing the existing experimental results, a bilinear model describing the frictional stress-slippage behavior of slab/base interface is adopted in the modeling. Expressions of shrinkage-induced stresses and displacements in the concrete slab lying on a supporting base are obtained. The model reflects the influences of concrete properties and slab/base in- terfacial friction characteristics, including free shrinkage behavior of concrete $\varepsilon_{c}$, elastic modulus of concrete $E_{c}$, steady friction stress of slab/base interface $\tau_{0}$ and the corresponding slippage value $\delta_{0}$, and dimensions of the slab. Due to the timedependent parameters of concrete, the model enables the prediction of the development of shrinkage-induced stress and displacement in the concrete slab with time since casting.

Model predictions with the experimentally obtained slab/ base friction stress-slippage relation of seven typical bases normally used in concrete pavement as inputs yield the following conclusions. The type of supporting bases significantly influences the magnitude of shrinkage-induced tensile stresses in concrete pavements. The shrinkage stress in concrete is not only a function of shrinkage strain, but also a function of characteristics of the slab/base interfacial friction stress. Under the same shrinkage strain value, the maximum difference between different bases on the shrinkage stress, for example, cement treated base and natural clay, may achieve several megapascals [Figs. 5(a and g)]. The stresses resulting from shrinkage in concrete pavements can be reduced by slab/base interface modifications.

The model can be used in both shrinkage cracking control and concrete pavement design. In addition, although the model is proposed primarily for studying the shrinkage-induced stress in concrete pavements, it can also be applied to similar problems such as temperature-induced stresses. The present analytical solution is applicable only to the case of restraint from the base.

\section{ACKNOWLEDGMENT}

This work was supported by a grant from the National Science Foundation (CMS-9872357) to the University of Michigan.

\section{REFERENCES}

Branson, D. E. (1977). Deformation of concrete structures, McGraw-Hill, New York.

Mehta, P. K. (1985). Concrete structures, properties and materials, Prentice-Hall, Englewood Cliffs, N.J.

Moody, E. D. (1998). "Transverse cracking distress in long-term pavement performance jointed concrete pavement sections." Transp. Res. Rec. 1629, Transportation Research Board, Washington, D.C., 6-12.

Mosley, W. H., and Bungey, J. H. (1990). Reinforced concrete design, Macmillan, U.K.

Neville, A. M. (1981). Properties of concrete, 3rd Ed., Pitman Publishing, Ltd., London.

Rozycki, D. K., and Rasmussen, R. O. (1998). “Assessment of slab-base interaction in PCC airfield pavements." Airport Fac.: Innovations for The Next Century, Proc., 25th Int. Air Transp. Conf., M. T. McNerney, ed., ASCE, New York, 403-422.

Stott, J. P. (1961). "Test on materials for use in sliding layers under concrete road slabs." Civ. Engrg., 56(663), 1297-1299, 1301; (669), 1466-1468; (655), 1603-1605.

Wesevich, J. W., McCullough, B. F., and Burns, N. H. (1987). "Stabilized subbase friction study for concrete pavements." Res. Rep. 495-1, Ctr. for Transp. Res., University of Texas at Austin, Austin, Tex.

Wimsatt, A. W., McCullough, B. F., and Burns, N. H. (1987). "Methods of analyzing and factors influencing frictional effects of subbases." Res. Rep. 495-2F, Ctr. for Transp. Res., University of Texas at Austin, Austin, Tex.

Yoder, E. J., and Witczak, M. W. (1975). Principles of pavement design, 2nd Ed., Wiley, New York. 